Research Paper

\title{
Role of the ERK $1 / 2$ Signaling Pathway in Osteogenesis of Rat Tendon-Derived Stem Cells in Normoxic and Hypoxic Cultures
}

\author{
Pei Li ${ }^{1}$, Yuan Xu ${ }^{2}$, Yibo Gan ${ }^{1}$, Lei Song 1, Chengmin Zhang 1, Liyuan Wang 1, Qiang Zhou ${ }^{\circledR}$ \\ 1. Department of Orthopedic Surgery, Southwest Hospital, Third Military Medical University, Chongqing, 400038, China; \\ 2. Department of Orthopedic Surgery, Xinqiao Hospital, Third Military Medical University, Chongqing, 400038, China. \\ $\bowtie$ Corresponding authors: E-mail: 83757870@qq.com (Yuan Xu); zq_tlh@163.com (Qiang Zhou).
}

() Ivyspring International Publisher. Reproduction is permitted for personal, noncommercial use, provided that the article is in whole, unmodified, and properly cited. See http://ivyspring.com/terms for terms and conditions.

Received: 2016.05.03; Accepted: 2016.06.25; Published: 2016.07.18

\begin{abstract}
Background: Ectopic ossification and increased vascularization are two common phenomena in the chronic tendinopathic tendon. The increased vascularization usually leads to an elevated local oxygen tension which is one of micro-environments that can influence differentiate status of stem cells.

Objective: This study aimed to investigate the osteogenesis capacity of rat tendon-derived stem cells TDSCs (rTDSCs) in normoxic and hypoxic cultures, and to study the role of ERK $1 / 2$ signaling pathway in this process.

Methods: rTDSCs were subjected to osteogenesis inductive culture in hypoxic $\left(3 \% \mathrm{O}_{2}\right)$ and normoxic $\left(20 \% \mathrm{O}_{2}\right)$ conditions. The inhibitor $\mathrm{U} 0126$ was added along with culture medium to determine the role of ERK $1 / 2$ signaling pathway. Cell viability, cell proliferation, alizarin red staining, alkaline phosphatase (AKP) activity, gene expression (ALP, osteocalcin, collagen I and RUNX2) and protein expression ( $\mathrm{p}$-ERK1/2 and RUNX2) of osteogenic-cultured rTSDCs were analyzed in this study.

Results: Hypoxic and normoxic culture had no effects on cell viability of rTDSCs, whereas the proliferation potential of rTDSCs was significantly increased in hypoxic culture. The osteogenesis capacity of rTDSCs in normoxic culture was significantly promoted compared with hypoxic culture, which was reflected by an increased alizarin red staining intensity, an elevated ALP activity, and the up-regulated gene (ALP, osteocalcin, collagen I and RUNX2) or protein (RUNX2) expression of osteogenic makers. However, the osteogenesis capacity of rTDSCs in both hypoxic and normoxic cultures was attenuated by the inhibitor $\mathrm{U} 0126$.

Conclusion: Normoxic culture promotes osteogenic differentiation of rTDSCs compared with the hypoxic culture, and the ERK $1 / 2$ signaling pathway is involved in this process.
\end{abstract}

Key words: tendinopathy, tendon-derived stem cells, hypoxic, normoxic, osteogenesis.

\section{Introduction}

Tendinopathy is a common painful tendon condition caused by overuse, mechanical injury or intrinsic degeneration [1-3]. Histologically, calcification is usually reported in some tendinopathies $[4,5]$, which leads to a failed self-healing and predisposes the diseased tendon to rupture [6]. Up to now, the etiopathogenesis for calcific tendinopathy remains unclear.

Tendon characterized as a kind of dense connective structures can lead to joint stabilization or joint movement through transferring mechanical load from muscle to bone $[7,8]$. Recently, a type of tendon-derived stem cell (TDSC) has been identified, which possesses the abilities of self-renewal and 
multi-lineage differentiation [9-11]. By differentiating into tenocytes, TDSCs play an important role in matrix homeostasis and tissue regeneration of the injured tendon [6, 12]. However, lots of abnormal repair outcomes are frequently observed in the pathological chronic tendinopathy, such as fibrocartilage-like tissue formation, lipid substance accumulation and ectopic ossification [13-15]. Recently, increasing evidence suggests that stem cells may also play a role in the pathological conditions [16, 17]. Several previous studies proposed that the erroneous differentiation of TDSCs to non-tenocytes caused by alterations of their surrounding micro-environments may contribute to the aberrant matrix remodeling and acquisition of non-tenocytes phynotype in the tendinopathic tendons [17, 18]. However, the potential mechanisms for the erroneous differentiation of TDSCs to non-tenocytes or other cellular phenotype are largely unknown. More direct evidences are needed to clarify this speculation.

Similar with other stem cells, oxygen tension is a local micro-environment surrounding TDSCs. In vivo, the oxygen tension within a certain tissue depends on the vascularization level and the inherent micro-environment type [19]. Under physiological conditions, the collagen-rich tendon has few blood vessels and thus a low oxygen level compared with other vascular-rich tissues [20]. By contrast, an increased vascular infiltration and capillary blood flow in the tendinopathic tendon are constantly reported previously [21-25], which may in turn lead to an elevated oxygen tension and thus an altered oxygen surrounding TDSCs. Generally, increased vascularization may be a protective response of tissue repair after injury. On another hand, differentiation of stem cells can also be regulated by oxygen tension [19, 26]. In other types of stem cells, oxygen tension alteration-induced changes in differentiation capacity are often reported during the past years [20, 27, 28]. Moreover, previous study demonstrated that osteogenic differentiation of bone mesenchymal stem cells (BMSCs) was promoted in normoxic culture. In light of the co-existence of ectopic ossification and increased vascular infiltration in the chronic tendinopathic tendon, we propose that the ectopic ossification may partly result from the erroneous osteogenic differentiation of TDSCs caused by increased local oxygen tension.

In the present study, we aimed to investigate the osteogenic differentiation capacity of rat TDSCs (rTDSCs) in hypoxic (3\%) culture and normoxic $(20 \%)$ culture. Because ERK1/2 pathway is a potential signaling pathway relating with differentiation of some stem cells, the potential role of ERK1/2 pathway was also determined by its pharmacological inhibitor
U0126. To achieve this purpose, cell viability, cell proliferation, AKP activity, alizarin red staining and expression of some osteogenic markers were evaluated in this study.

\section{Materials and methods}

\section{Ethical statement}

All animal experiments in this study were approved by Ethics Committee at Southwest Hospital affiliated to the Third Military Medical University [SYXK (YU) 2012-0012].

\section{Isolation and preparation of rTDSCs}

rTDSCs were isolated from the achilles tendon of twelve healthy rats (male, 4-5 weeks old) as described previously $[29,30]$. Briefly, after rats were sacrificed with carbon dioxide, their bilateral achilles tendons were separated. Then, the tendon sheaths and paratendons were further removed. Thereafter, the tendons were cut into small pieces (approximately 2 $\mathrm{mm} \times 2 \mathrm{~mm}$ ) and digested with phosphate buffered saline (PBS) supplemented with $0.3 \%$ type I collagenase (Sigma) and $0.4 \%$ neutral protease (Roche) at $37^{\circ} \mathrm{C}$ for $50-60 \mathrm{~min}$. After digestion and centrifugation (500 g, $15 \mathrm{~min}$ ), cell pellets were collected and re-suspended in DMEM/F12 medium (Hyclone) containing 20\% fetal bovine serum (FBS, Gibco) under standard conditions $\left(37^{\circ} \mathrm{C}, 20 \% \mathrm{O}_{2}\right.$ and $5 \% \mathrm{CO}_{2}$ ). After 8-10 days, TDSCs were collected by local trypsin digestion of individual cell colonies under a light microscopy (Olympus, BX51) and defined as the passage 0 rTDSCs according to previous study [29]. Then, the isolated rTDSCs were sub-cultured and passaged after reaching 80\%-90\% confluence. Previously, we demonstrated that passage 3 rTDSCs displayed a good colongenicity and vigorous differentiation capacity [29]. Hence, we mainly used the passage 3 rTDSCs in each experiment in the present study.

\section{Hypoxic and normoxic osteoinductive culture of rTDSCs}

The P3 rTDSCs were cultured in Osteogenic Differentiation Medium (Cyagen Biosciences Inc) and incubated in a hypoxic $\left(3 \% \mathrm{O}_{2}\right)$ incubator or a normoxic $\left(20 \% \mathrm{O}_{2}\right)$ incubator (Thermo Scientific). To investigate the role of ERK1/2 signaling pathway, the inhibitor U0126 (10 $\mu \mathrm{M}$, Beyotime, China) was added along with the medium throughout the experiment. Culture medium was refreshed every 3 days. To accurately maintain the oxygen tension in the hypoxic and normoxic cultures as much as possible, a rapid and timely gas injection process was performed after exchanging the culture medium. Because no study reported the measurement of oxygen tension in 
human normal tendon even though the tendon milieu is estimated to be hypoxic, the hypoxic and normoxic cultures were designed according to previous studies $[19,20,31,32]$.

\section{Cell viability}

rTDSCs were seeded in 6-well plate $\left(4 \times 10^{3}\right.$ cells/well) and osteogenic-cultured in the designed oxygen tension conditions. On days 1, 4 and 7, cell viability of rTDSCs was analyzed with a LIVE/DEAD Viability/Cytotoxicity Assay Kit (Invitrogen) according to the instructions. Briefly, after washing with PBS for 2-3 times, rTDSCs in hypoxic and nomorxic cultures were incubated with fluorescent working solution (calcein AM: $2 \mu \mathrm{M}$; EthD-1: $4 \mu \mathrm{M}$ ) for 40 minutes at room temperature. Then, the live or dead rTDSCs in each group were viewed under a fluorescence microscopy (Olympus IX71). Quantification of cell viability was performed using the Image-Pro Plus software (Version 5.1, Media Cybernetics, Inc.)

\section{Cell proliferation assay}

On days 1, 4 and 7, rTDSCs proliferation potential was evaluated with a Cell Counting Kit-8 (CCK-8, Beyotime, China). Briefly, after rTDSCs (seeded in 12-well plate, $2 \times 10^{3}$ cells/well) were incubated with fresh medium containing CCK-8 solution for 2 hours, $200 \mu \mathrm{L}$ supernatant was used to measure the absorbance at $450 \mathrm{~nm}$ wavelength using an automatic micro-plate reader (Bio-rad).

\section{Alizarin red staining assay}

rTDSCs (seeded in 10-cm diameter dish, $10 \times 10^{3}$ cells/dish) were osteogenic-cultured in medium with or without inhibitor U0126 under different oxygen tension conditions. After 21 days of osteogenic differentiation, the culture medium was removed and the rTDSCs were sequentially fixed with $3 \mathrm{ml} 4 \%$ paraformaldehyde for 30 minutes, rinsed with PBS for 3 times and stained with alizarin red working solution (Cyagen Biosciences Inc.) for 5-8 minutes. Finally, rTDSCs were observed under a light microscopy (Olympus BX51). Quantification of alizarin red staining intensity was performed using the Image-Pro Plus software (Version 5.1, Media Cybernetics, Inc.)

Table 1. Primers of target genes.

\begin{tabular}{lll}
\hline Gene & Forward $\left(5^{\prime}-3^{\prime}\right)$ & Reverse $\left(5^{\prime}-3^{\prime}\right)$ \\
\hline$\beta$-actin & ACCCCGTGCTGCTGACCGAG & TCCCGGCCAGCCAGGTCCA \\
osteocalcin & CGGCGCTACCTCAACAATGG & GTCCATACTTTCGAGGCAGAGAG \\
Collagen I & CATCGTGGCTTCTCTGGTC & ACCGTTGAGTCCATCTTTGC \\
ALP & CCCGAGTGCTTTGTGTGTGCTG & CCGCCGGTGTTCGTGTGTG \\
RUNX2 & GGGCAGATGGGGAACTGTG & GGTTTGCTACTGGGTGGGTTTC \\
\hline
\end{tabular}

Alkaline phosphatase (AKP) activity detection

rTDSCs were seeded in 10-cm diameter dishes $\left(10 \times 10^{3}\right.$ cells/dish) and osteogenic-cultured for 14 or 21 days. Then, rTDSCs were incubated with lysis buffer $(200 \mu \mathrm{L}$, Beyotime, China) and centrifuged (15, $000 \mathrm{r} / \mathrm{min}, 15 \mathrm{~min}$ ) to collect lysis supernatant, protein concentration was measured with BCA Kit (Beyotime, China). Then, AKP activity was detected with an Alkaline Phosphatase (AKP) Kit (Nanjing Jiancheng Bioengineering Institute, China) according to the manufacture's instructions.

\section{Real-time polymerase chain reaction (PCR) analysis}

Gene expression of several osteogenic markers (ALP, osteocalcin, collagen I and RUNX2) was analyzed by real-time PCR as described [29]. Briefly, rTDSCs were seeded in 10-cm diameter dishes $(10 \times$ $10^{3}$ cells/dish) and osteogenic-cultured under different oxygen tension conditions. On days 14 and 21, total RNA was extracted with Tripure Isolation Reagent (Roche) and reverse-transcripted into cDNA with a reverse transcription kit (Roche). Then, the reaction system containing CDNA, primers and SYBR Green Mix (DONGSHENG BIOTECH, China) was subjected to a real-time PCR machine (CFX96 Real-Time System, Bio-rad). Primers of target genes were showed in the Table 1 . $\beta$-actin was used as the reference gene and the P3 rTDSCs collected immediately were used as controls. The relative gene expression of target genes was expressed as $2-\Delta \Delta \mathrm{Ct}$.

\section{Western blotting analysis}

Protein expression of ERK1/2, p-ERK1/2 and osteogenic maker (RUNX2) was analyzed by Western blotting assay. Briefly, after total protein of rTDSCs osteogenic-cultured in different oxygen tension conditions for 21 days was extracted with RIPA solution (Beyotime, China), protein samples were subjected to SDS-PAGE and transferred to PVDF membrane (Roche). Then, the PVDF membrane was blocked with $5 \%$ bovine serum albumin (BSA) and incubated with primary antibodies (ERK1/2, 1:500, sc-292838, Santa Cruz; p-ERK1/2, 1:500, sc-101761, Santa Cruz; RUNX2, 1:500, sc-390351, Santa Cruz; $\beta$-actin, 1:1000, 60008-1-Ig, Proteintech) overnight at $4^{\circ} \mathrm{C}$ and HRP-conjugated secondary antibodies (ZSGB-BIO, China) for 2 hours at room temperature. Finally, protein bands on the PVDF membrane were visualized using the SuperSignal West Pico Trial Kit (Thermo) and analyzed using the Image J software (National Institutes of Health, USA). 

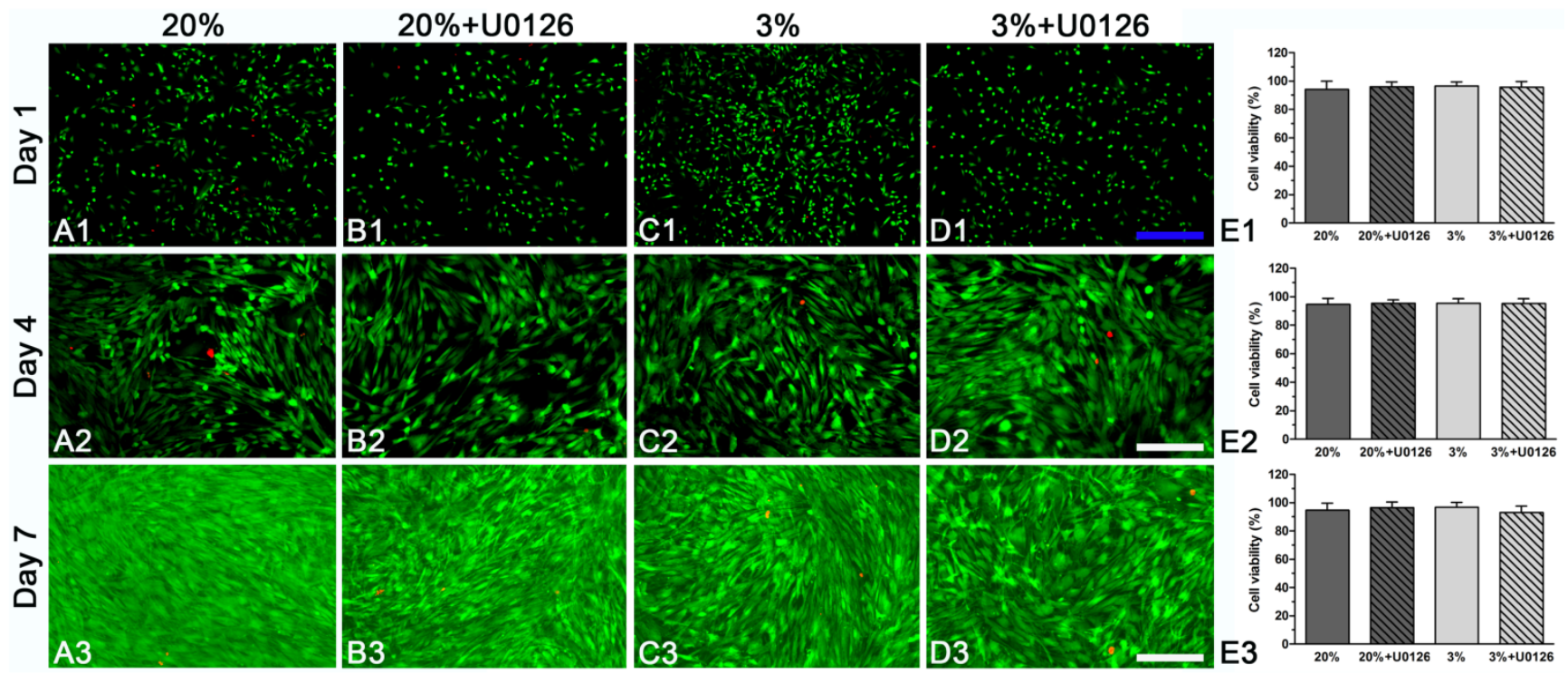

Figure 1: Cell viability analysis of rat tendon-derived stem cells (rTDSCs) in hypoxic and normoxic cultures on days 1, 4 and 7 . The live and dead cells were stained with green fluorescence and red fluorescence, respectively. Magnification: A1-D1, 40x; A2-D2 and A3-D3, 100x. n=3.

\section{Statistics}

All numerical data are expressed as mean \pm SD and analyzed by the SPSS 13.0 software. Each experiment in this study was performed in triplicate. When homogeneity test for variance was completed, comparisons between normoxic culture and hypoxic culture, between normoxic culture without U0126 treatment and normoxic culture with U0126 treatment, and between hypoxic culture without U0126 and hypoxic culture with U0126 treatment were analyzed by Independent-Samples $\mathrm{T}$ test. A statistical difference was indicated when p-value $<0.05$.

\section{Results}

\section{Cell viability}

Both in hypoxic and normoxic cultures, osteogenic-cultured rTDSCs remained viable on days 1, 4 and 7 (Figure 1A1-3, C1-3). Generally, there were no differences in cell viability between hypoxic culture and normoxic culture. Inhibition of ERK1/2 signaling pathway had no effects on cell viability in hypoxic and normoxic cultures (Figure 1B1-3, D1-3).

\section{Cell proliferation}

Throughout the 7 days of culture, osteogenic-cultured rTDSCs showed a consistent proliferation potential both in the hypoxic and normoxic cultures (Figure 2). Although there was no significant difference in cell proliferation between the hypoxic and normoxic cultures at day 1 , proliferation potential in hypoxic culture was significantly increased compared with normoxic culture at days 4 and 7. Additionally, the inhibitor U0126 obviously attenuated cell proliferation in hypoxic and normoxic cultures on days 4 and 7 .

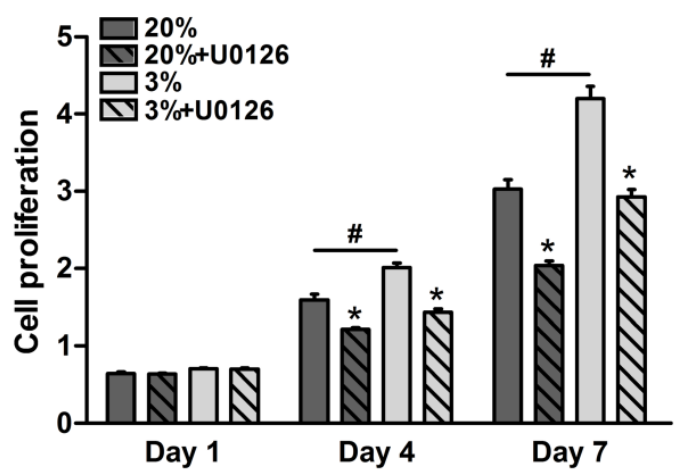

Figure 2: Cell proliferation potential of rat tendon-derived stem cells (rTDSCs) in hypoxic and normoxic cultures on days 1, 4 and 7. Date are expressed as mean $\pm S D, n=3$. \#: Indicates a significant difference between hypoxic and normoxic cultures without addition of inhibitor U0126. *: Indicates a significant difference associated with U0126 treatment in hypoxic culture or normoxic culture.

\section{Alizarin red staining}

A stronger alizarin red staining intensity was observed in normoxic culture compared with hypoxic culture (Figure 3). However, inhibition of ERK1/2 signaling pathway in hypoxic or normoxic culture significantly decreased the staining intensity (Figure $3)$.

\section{AKP activity}

In normoxic culture, AKP activity of osteogenic-cultured rTDSCs was significantly increased compared with that in hypoxic culture on days 14 and 21 (Figure 4). Either in hypoxic or 
normoxic culture, AKP activity was decreased when the ERK1/2 signaling pathway was inhibited by inhibitor U0126.

\section{Gene expression}

Genes of osteogenic maker were differently expressed in hypoxic and normoxic cultures (Figure 5). In normoxic culture, expression of ALP, osteocalcin, collagen I and RUNX2 was all up-regulated compared with that in hypoxic culture on days 14 and 21. However, addition of U0126 in either hypoxic or normoxic culture inhibited gene expression of these osteogenic markers (Figure 5).

\section{Protein expression}

In normoxic culture, protein expression of p-ERK1/2 or RUNX2 was up-regulated compared with hypoxic culture (Figure 6). When the expression of p-ERK1/2 was inhibited by inhibitor U0126 in normoxic and hypoxic cultures, expression of RUNX2 was simultaneously down-regulated (Figure 6).

\section{Discussion}

Ectopic ossification is commonly found in the chronic tendinopathic tendon [33]. Currently, the mechanism underlying this pathological process remains unknown. Apart from the ectopic ossification, oxygen tension may be also elevated due to the increased vascular infiltration [24]. Considering that TDSCs can erroneously differentiate into non-tenocytes due to the altered micro-environments and thus play a role in pathological conditions, we performed this study to investigate the osteogenesis capacity of rTDSCs in the hypoxic and normoxic cultures. Our results showed that rTDSCs remained viable both in hypoxic and normoxic cultures and displayed a stronger proliferation potential in hypoxic culture. Specially, rTDSCs in normoxic culture possessed a promoted osteogenesis capacity regarding alizarin red staining, AKP activity, gene expression of osteogenesis-related markers (ALP, osteocalcin, collagen I and RUNX2) and protein expression of RUNX2. Additionally, we also found that inhibition of ERK1/2 signaling pathway could attenuate the osteogenesis potential of rTDSCs (summarized in the Figure 7). These findings demonstrated that oxygen tension is an important micro-environment for regulating osteogenic differentiation of TDSCs, and also indicated that ERK1/2 signaling pathway is involved in this regulatory process.
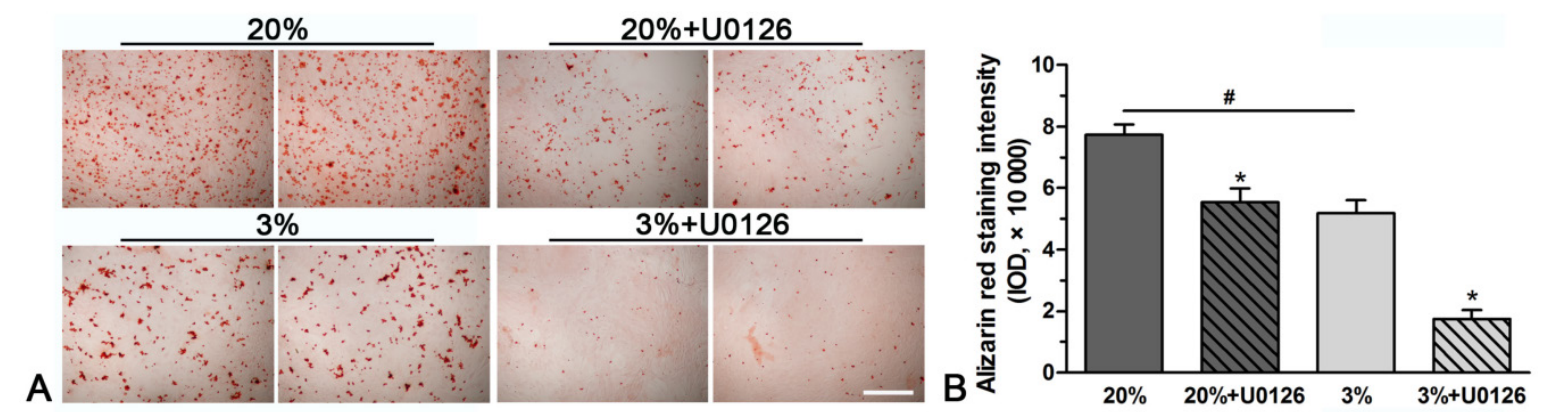

Figure 3: Representative photomicrographs and quantification of alizarin red staining of rat tendon-derived stem cells (rTDSCs) in hypoxic and normoxic cultures on day 21 . Magnification: $100 x . n=3$.

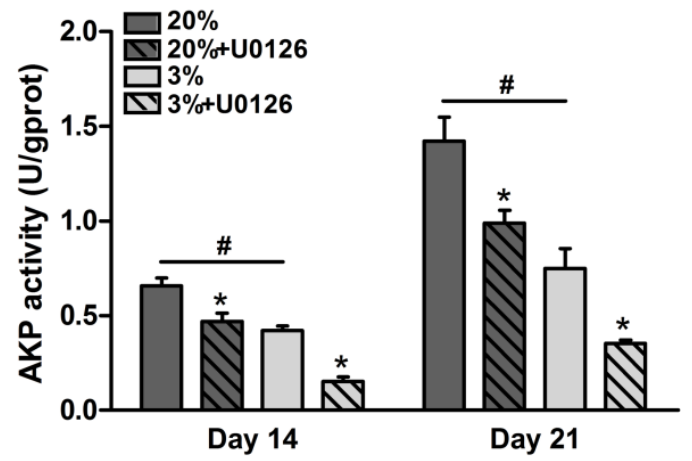

Figure 4: Alkaline phosphatase (AKP) activity of rat tendon-derived stem cells (rTDSCs) in hypoxic and normoxic cultures on days 14 and 21 . Date are expressed as mean $\pm S D, n=3$. \#: Indicates a significant difference between hypoxic and normoxic cultures without addition of inhibitor $\cup 0126$. *: Indicates a significant difference associated with U0126 treatment in hypoxic culture or normoxic culture. 


\section{RUNX2}

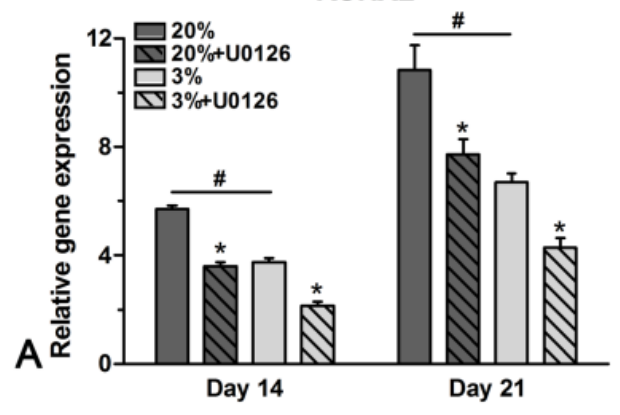

Collagen I

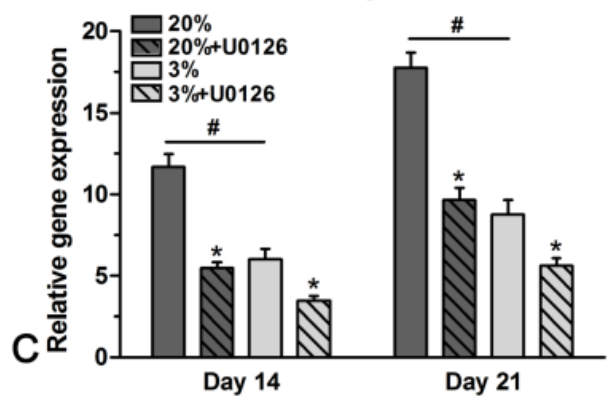

Osteocalcin

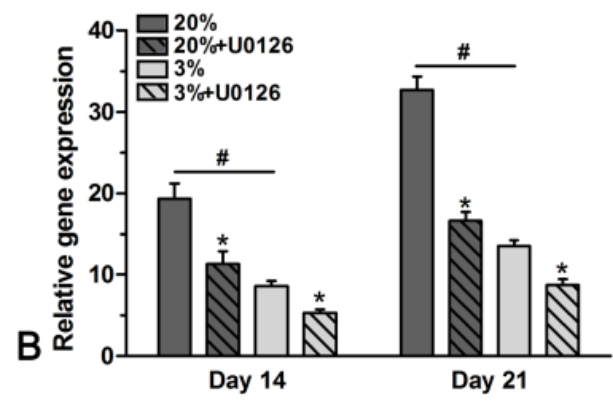

ALP

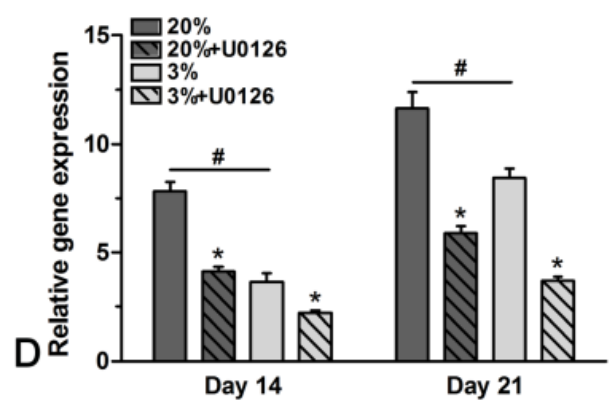

Figure 5: Real-time PCR analysis of rat tendon-derived stem cells (rTDSCs) in hypoxic and normoxic cultures on days 14 and 21 . Date are expressed as mean \pm SD, $\mathrm{n}=3$. \#: Indicates a significant difference between hypoxic and normoxic cultures without addition of inhibitor U0126. *: Indicates a significant difference associated with U0126 treatment in hypoxic culture or normoxic culture.

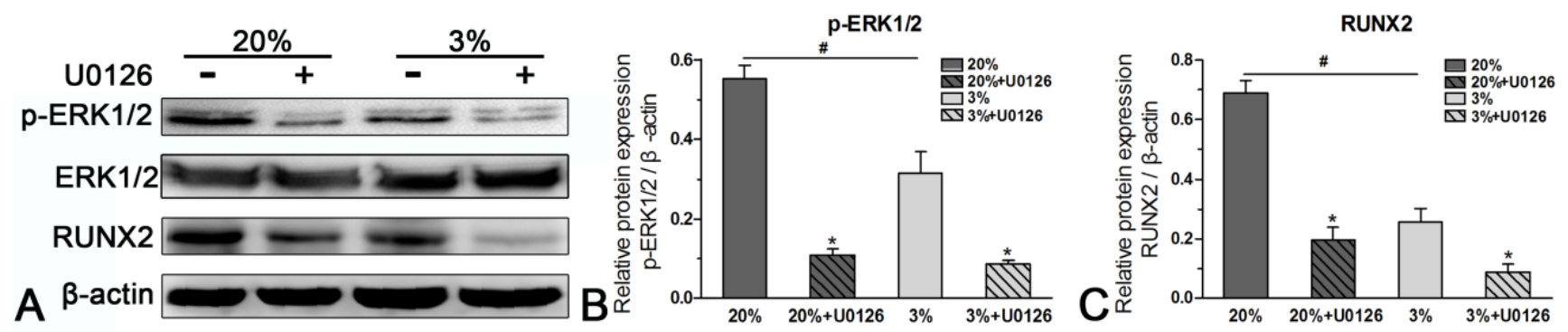

Figure 6: Western blotting analysis of rat tendon-derived stem cells ( $r T D S C s)$ in hypoxic and normoxic cultures on day 21 . Date are expressed as mean \pm SD, $n=3$. \#: Indicates a significant difference between hypoxic and normoxic cultures without addition of inhibitor U0126. *: Indicates a significant difference associated with U0126 treatment in hypoxic culture or normoxic culture.

TDSCs are stem cells residing in tendon tissue. Similar with other types of stem cells, TDSCs interplay with the local micro-environment to participate in tendon healing and tendon matrix remodeling after injury [6]. In tendon, mechanical loading, matrix composition, biological factors and some other physiological factors are typical micro-environments which can regulate biological responses of TDSCs [34]. There are also some evidences that aberrant micro-environments can lead to abnormal functions of stem cells and ultimately pathological diseases [6, 17, 34]. In chronic tendinopathic tendon, ossification and increased blood vessels are two common pathological features. Hence, the raised oxygen tension resulted from the increased blood vessels may lead to erroneous osteogenic differentiation of TDSCs and thus some ossification tissues in the diseased tendon. In line with us, aberrant osteogenic differentiation of stem cells is also previously reported in other tissues, such as arterial calcification and skin calcification $[35,36]$.

Oxygen tension is low in the healthy tendon since it has a low blood flow, while oxygen tension may tend to rise in the tendinopathic tendon because of the increased vascular infiltration [34, 37]. In this study, we found that rTDSCs have an increased alizarin red staining intensity under normoxic condition compare with that under hypoxic condition. Similarly, the results of ALP activity assay also showed a similar trend to that observed in alizarin red staining. These findings indicate that the osteogenesis capacity of rTDSCs in normoxic culture is promoted compared with that in hypoxic culture. In line with us, a previous study also indicated that human TDSCs 
have a reduced osteogenic differentiation potential but an increased proliferation capacity in hypoxic $(2 \%)$ culture [32]. Additionally, it is also reported that osteogenic differentiation of bone mesenchymal stem cells (BMSCs) was also attenuated in hypoxic culture $[19,38]$. However, a previous study by Zhang et al. showed that osteogenic differentiation of human TDSCs in hypoxic (5\%) culture was increased compared with that in normoxic $(20 \%)$ culture [20]. We speculate there are several possible factors that may be responsible for this discrepancy, such as the different control way of oxygen tension in the incubator, the different initial status and source of TDSCs, and different experimental conditions [20]. Nevertheless, all these studies indicate that oxygen tension is an important factor for regulating osteogenesis of TDSCs.

Various osteogenesis markers, either for the early stage or the late stage, had been identified previously, such as ALP, collagen I, RUNX2, osteonectin, osteocalcin and osteopontin [39]. In the present study, we investigated expression of these osteogenic makers from gene level or protein level. We found that gene expression of ALP, osteocalcin, RUNX2 and collagen I as well as protein expression of RUNX2 are all up-regulated in the normoxic culture compared with hypoxic osteogenic culture. This suggests again that osteogenesis capacity of rTDSC in normoxic culture is promoted compared with that in

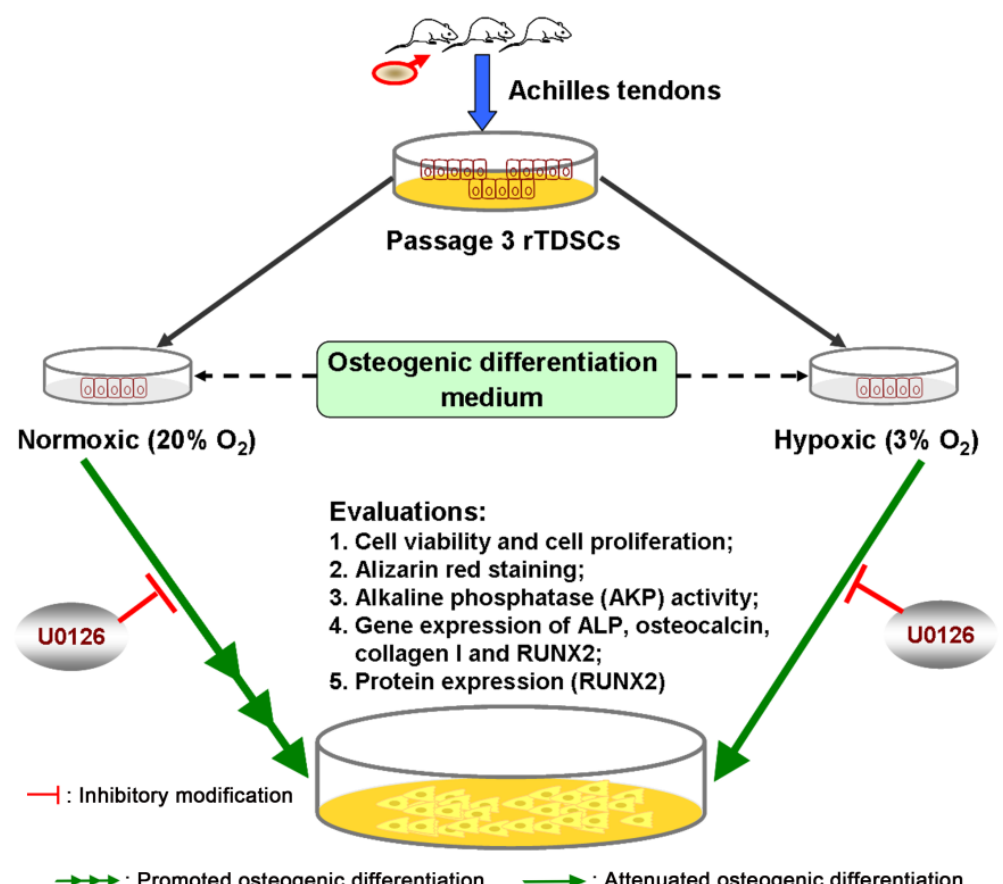

Figure 7: A brief graphic abstract of this study. Rat tendon-derived stem cells (rTDSCs) were cultured in normoxic $\left(20 \% \mathrm{O}_{2}\right)$ and hypoxic $\left(3 \% \mathrm{O}_{2}\right)$ cultures. Osteogenesis capacity of rTDSCs in normoxic culture was promoted compared with that in hypoxic culture, whereas inhibition of ERK1/2 signaling pathway attenuated osteogenesis of rTDSCs both in normoxic and hypoxic cultures. hypoxic culture. Additionally, this also indirectly implies that the ossification tissue in the chronic tendinopathic tendon is related to osteogenesis of TDSCs caused by alteration of local micro-environment, such as the elevated oxygen tension caused by the increase in vascularization.

ERK1/2 signaling pathway is a branch of MAPK pathways which are involved in many cell bioactivities including cell proliferation, cell apoptosis and cell differentiation [40]. Previously, ERK1/2 signaling pathway had been reported to participate in inhibiting the osteogenic differentiation of BMSC under hypoxic condition [38]. In this study, activation of ERK1/2 signaling pathway in rTDSCs in normoxic culture was more obvious than that in hypoxic culture. Moreover, when ERK1/2 signaling pathway was inhibited by inhibitor U0126, osteogenic activity of rTDSCs regarding alizarin red staining intensity, ALP activity and expression of the designed osteogenesis markers was simultaneously decreased. These results indicate that the ERK1/2 signaling pathway is involved in the effects of altered oxygen tension on osteogenesis capacity of rTDSCs. Consistent with us, activation of ERK1/2 signaling pathway is also previously reported to participate in the osteogenesis of other types of stem cells, such as BMSCs, Periodontal ligament stem cells and induced pluripotent stem cells [41-43].

Previous studies demonstrated that oxygen tension can affect cell viability and cell proliferation of stem cells. In this study, no differences in cell viability of osteogenic-cultured rTDSCs were found between hypoxic culture and normoxic culture, indicating that rTDSCs can remain viable in either hypoxic culture or normoxic culture. However, proliferation capacity of the rTDSCs in hypoxic culture was increased compared with that in normoxic culture. This finding confirmed previous stem cells-related studies which demonstrated that the stemness of stem cells is better maintained in hypoxic culture [19, $32,44]$. Apart from this, we also found that blocking the ERK1/2signaling pathway in hypoxic or normoxic culture inhibited cell proliferation of rTDSCs, whereas the cell viability was not influenced. This indicates that ERK1/2 signaling pathway may affect cell proliferation but not cell viability of rTDSCs in different oxygen tension conditions.

This study also has several limitations. First, an in vivo animal model is not used to verify the results from the in vitro cell 
culture system. Second, erroneous osteogenic differentiation of TDSCs may be resulted from a combination of several factors including elevated oxygen tension, inflammation, mechanical overloading and alterations in extracellular matrix [34]. However, we just studied the effects of single factor on osteogenesis capacity of rTDSCs in this study. Third, because there are no reports about the measurement of exact value of oxygen extension in human tendon under physiological and pathological conditions, the oxygen tension values of hypoxic and normoxic cultures in this study were designed according to previous studies [20, 32]. Hence, the oxygen tension parameters used in this study may differ from the actual oxygen tension in human tendon under physiological and pathological conditions.

Taken together, we can draw the conclusion that osteogenesis capacity of rTSDCs in the normoxic culture was increased compared with that in the hypoxic culture, and ERK1/2 phosphorylation may participate in this regulatory process. This study will contribute to further understanding of the mechanism behind the ectopic ossification in the tendinopathic tendon and ultimately the development of effective clinical treatment for it.

\section{Acknowledgments}

We appreciate the founding from the National Natural Science Foundation of China (NSFC 81272029 and NSFC 81027005), Science and Technology Achievement Transformation Fund of the Third Military Medical University (2011XZH006).

\section{Conflicts of Interest}

The authors report no conflicts of interest.

\section{References}

1. Vora AM, Myerson MS, Oliva F, Maffulli N. Tendinopathy of the main body of the Achilles tendon. Foot and ankle clinics. 2005; 10: 293-308.

2. Weinreb JH, Sheth C, Apostolakos J, McCarthy MB, Barden B, Cote MP, et al. Tendon structure, disease, and imaging. Muscles Ligaments Tendons J. 2014; 4: 66-73.

3. Way L, Scutt N, Scutt A. Cytocentrifugation: a convenient and efficient method for seeding tendon-derived cells into monolayer cultures or 3-D tissue engineering scaffolds. Cytotechnology. 2011; 63: 567-79.

4. Kannus P. Structure of the tendon connective tissue. Scand J Med Sci Sports. 2000; 10: 312-20.

5. Oliva F, Via AG, Maffulli N. Physiopathology of intratendinous calcific deposition. BMC medicine. 2012; 10: 95.

6. Lui PP, Chan KM. Tendon-derived stem cells (TDSCs): from basic science to potential roles in tendon pathology and tissue engineering applications. Stem Cell Rev. 2011; 7: 883-97.

7. Birch HL. Tendon matrix composition and turnover in relation to functional requirements. Int J Exp Pathol. 2007; 88: 241-8.

8. Hodgson RJ, O'Connor PJ, Grainger AJ. Tendon and ligament imaging. Br J Radiol. 2012; 85: 1157-72.

9. Bi Y, Ehirchiou D, Kilts TM, Inkson CA, Embree MC, Sonoyama W, et al. Identification of tendon stem/progenitor cells and the role of the extracellular matrix in their niche. Nat Med. 2007; 13: 1219-27.

10. Salingcarnboriboon R, Yoshitake H, Tsuji K, Obinata M, Amagasa T, Nifuji A, et al. Establishment of tendon-derived cell lines exhibiting pluripotent mesenchymal stem cell-like property. Exp Cell Res. 2003; 287: 289-300.
11. Scutt N, Rolf CG, Scutt A. Glucocorticoids inhibit tenocyte proliferation and Tendon progenitor cell recruitment. Journal of orthopaedic research : official publication of the Orthopaedic Research Society. 2006; 24: 173-82.

12. Zhang J, Wang JH. Characterization of differential properties of rabbit tendon stem cells and tenocytes. BMC Musculoskelet Disord. 2010; 11: 10.

13. Riley GP, Harrall RL, Constant CR, Cawston TE, Hazleman BL. Prevalence and possible pathological significance of calcium phosphate salt accumulation in tendon matrix degeneration. Ann Rheum Dis. 1996; 55: 109-15.

14. Maffulli N, Reaper J, Ewen SW, Waterston SW, Barrass V. Chondral metaplasia in calcific insertional tendinopathy of the Achilles tendon. Clin J Sport Med. 2006; 16: 329-34.

15. Fenwick S, Harrall R, Hackney R, Bord S, Horner A, Hazleman B, et al. Endochondral ossification in Achilles and patella tendinopathy. Rheumatology (Oxford). 2002; 41: 474-6.

16. Caplan AI, Bruder SP. Mesenchymal stem cells: building blocks for molecular medicine in the 21st century. Trends Mol Med. 2001; 7: 259-64.

17. Rui YF, Lui PP, Chan LS, Chan KM, Fu SC, Li G. Does erroneous differentiation of tendon-derived stem cells contribute to the pathogenesis of calcifying tendinopathy? Chin Med J (Engl). 2011; 124: 606-10.

18. Zhang J, Wang JH. Mechanobiological response of tendon stem cells: implications of tendon homeostasis and pathogenesis of tendinopathy. Journal of orthopaedic research : official publication of the Orthopaedic Research Society. 2010; 28: 639-43.

19. Fehrer C, Brunauer R, Laschober G, Unterluggauer H, Reitinger S, Kloss F, et al. Reduced oxygen tension attenuates differentiation capacity of human mesenchymal stem cells and prolongs their lifespan. Aging Cell. 2007; 6: 745-57.

20. Zhang J, Wang JH. Human tendon stem cells better maintain their stemness in hypoxic culture conditions. PLoS One. 2013; 8: e61424.

21. Knobloch K, Kraemer R, Lichtenberg A, Jagodzinski M, Gossling T, Richter M, et al. Achilles tendon and paratendon microcirculation in midportion and insertional tendinopathy in athletes. Am J Sports Med. 2006; 34: 92-7.

22. Alfredson H, Ohberg L, Forsgren S. Is vasculo-neural ingrowth the cause of pain in chronic Achilles tendinosis? An investigation using ultrasonography and colour Doppler, immunohistochemistry, and diagnostic injections. Knee Surg Sports Traumatol Arthrosc. 2003; 11: 334-8.

23. Andarawis-Puri N, Flatow EL, Soslowsky LJ. Tendon basic science: Development, repair, regeneration, and healing. Journal of orthopaedic research : official publication of the Orthopaedic Research Society. 2015; 33: $780-4$.

24. Xu Y, Murrell GA. The basic science of tendinopathy. Clin Orthop Relat Res. 2008; 466: 1528-38

25. Knobloch K. The role of tendon microcirculation in Achilles and patellar tendinopathy. Journal of orthopaedic surgery and research. 2008; 3: 18.

26. Kawasaki T, Sumita Y, Egashira K, Ohba S, Kagami H, Tran SD, et al. Transient Exposure to Hypoxic and Anoxic Oxygen Concentrations Promotes Either Osteogenic or Ligamentogenic Characteristics of PDL Cells. Biores Open Access. 2015; 4: 175-87.

27. Lennon DP, Edmison JM, Caplan AI. Cultivation of rat marrow-derived mesenchymal stem cells in reduced oxygen tension: effects on in vitro and in vivo osteochondrogenesis. J Cell Physiol. 2001; 187: 345-55.

28. Berniakovich I, Giorgio M. Low oxygen tension maintains multipotency, whereas normoxia increases differentiation of mouse bone marrow stromal cells. Int J Mol Sci. 2013; 14: 2119-34.

29. Xu Y, Wang Q, Li Y, Gan Y, Li P, Li S, et al. Cyclic Tensile Strain Induces Tenogenic Differentiation of Tendon-Derived Stem Cells in Bioreactor Culture. Biomed Res Int. 2015; 2015: 790804.

30. Ni M, Lui PP, Rui YF, Lee YW, Lee YW, Tan Q, et al. Tendon-derived stem cells (TDSCs) promote tendon repair in a rat patellar tendon window defect model. Journal of orthopaedic research : official publication of the Orthopaedic Research Society. 2012; 30: 613-9.

31. Fan L, Liu R, Li J, Shi Z, Dang X, Wang K. Low oxygen tension enhances osteogenic potential of bone marrow-derived mesenchymal stem cells with osteonecrosis-related functional impairment. Stem Cells Int. 2015; 2015: 950312.

32. Lee WY, Lui PP, Rui YF. Hypoxia-mediated efficient expansion of human tendon-derived stem cells in vitro. Tissue Eng Part A. 2012; 18: 484-98.

33. Oliva F, Via AG, Maffulli N. Calcific tendinopathy of the rotator cuff tendons. Sports Med Arthrosc. 2011; 19: 237-43

34. Lui PP. Identity of tendon stem cells--how much do we know? J Cell Mol Med. 2013; 17: 55-64.

35. Speer MY, Yang HY, Brabb T, Leaf E, Look A, Lin WL, et al. Smooth muscle cells give rise to osteochondrogenic precursors and chondrocytes in calcifying arteries. Circ Res. 2009; 104: 733-41.

36. Kim SY, Choi HY, Myung KB, Choi YW. The expression of molecular mediators in the idiopathic cutaneous calcification and ossification. J Cutan Pathol. 2008; 35: 826-31.

37. Benjamin M, Ralphs JR. Tendons and ligaments--an overview. Histol Histopathol. 1997; 12: 1135-44.

38. Wang Y, Li J, Wang Y, Lei L, Jiang C, An S, et al. Effects of hypoxia on osteogenic differentiation of rat bone marrow mesenchymal stem cells. Mol Cell Biochem. 2012; 362: 25-33.

39. Vater C, Kasten P, Stiehler M. Culture media for the differentiation of mesenchymal stromal cells. Acta Biomater. 2011; 7: 463-77. 
40. Liu L, Zhang H, Sun L, Gao Y, Jin H, Liang S, et al. ERK/MAPK activation involves hypoxia-induced MGr1-Ag/37LRP expression and contributes to apoptosis resistance in gastric cancer. Int J Cancer. 2010; 127: 820-9.

41. Bai $\mathrm{B}, \mathrm{He} \mathrm{J}, \mathrm{Li}$ YS, Wang XM, Ai HJ, Cui FZ. Activation of the ERK1/2 signaling pathway during the osteogenic differentiation of mesenchymal stem cells cultured on substrates modified with various chemical groups. Biomed Res Int. 2013; 2013: 361906

42. Ye G, Li C, Xiang X, Chen C, Zhang R, Yang X, et al. Bone morphogenetic protein-9 induces PDLSCs osteogenic differentiation through the ERK and p38 signal pathways. Int J Med Sci. 2014; 11: 1065-72.

43. Zhang P, Dai Q, Ouyang N, Yang X, Wang J, Zhou S, et al. Mechanical Strain Promotes Osteogenesis of BMSCs from Ovariectomized Rats via the ERK1/2 but not p38 or JNK-MAPK Signaling Pathways. Curr Mol Med. 2015; 15: 780-9.

44. Yoshida Y, Takahashi K, Okita K, Ichisaka T, Yamanaka S. Hypoxia enhances the generation of induced pluripotent stem cells. Cell Stem Cell. 2009; 5: 237-41. 